\title{
Patterns of virulence factor expression and antimicrobial resistance in Achromobacter xylosoxidans and Achromobacter ruhlandii isolates from patients with cystic fibrosis
}

\author{
R. H. V. PEREIRA ${ }^{1}$, R. S. LEÃO ${ }^{1}$, A. P. CARVALHO-ASSEF ${ }^{2}$, \\ R. M. ALBANO ${ }^{3}$, E. R. A. RODRIGUES ${ }^{1}$, M. C. FIRMIDA ${ }^{1}$, T. W. FOLESCU ${ }^{4}$, \\ M. C. PLOTKOWSKI ${ }^{1}$, V. G. BERNARDO ${ }^{3}$ AND E. A. MARQUES ${ }^{1 *}$ \\ ${ }^{1}$ Departamento de Microbiologia, Imunologia e Parasitologia, Faculdade de Ciências Médicas, Universidade do \\ Estado do Rio de Janeiro, Brazil \\ ${ }^{2}$ Laboratório de Pesquisa em Infecção Hospitalar, Instituto Oswaldo Cruz, Rio de Janeiro, Brazil \\ ${ }^{3}$ Departamento de Bioquímica, Instituto de Biologia Roberto Alcântara Gomes, Universidade do Estado do Rio de \\ Janeiro, Brazil \\ ${ }^{4}$ Instituto Fernandes Figueira, Fundação Instituto Oswaldo Cruz, Rio de Janeiro, Brazil
}

Received 14 June 2016; Final revision 14 October 2016; Accepted 14 October 2016;

first published online 22 November 2016

\section{SUMMARY}

Achromobacter spp. are opportunistic pathogens increasingly recovered from adult patients with cystic fibrosis (CF). We report the characterization of 122 Achromobacter spp. isolates recovered from $39 \mathrm{CF}$ patients by multilocus sequence typing, virulence traits, and susceptibility to antimicrobials. Two species, A. xylosoxidans $(77 \%)$ and A. ruhlandii $(23 \%)$ were identified. All isolates showed a similar biofilm formation ability, and a positive swimming phenotype. By contrast, $4 \cdot 3 \%$ and $44 \cdot 4 \%$ of $A$. xylosoxidans and A. ruhlandii, respectively, exhibited a negative swarming phenotype, making the swimming and swarming abilities of $A$. xylosoxidans significantly higher than those of $A$. ruhlandii. A. xylosoxidans isolates from an outbreak clone also exhibited significantly higher motility. Both species were generally susceptible to ceftazidime, ciprofloxacin, imipenem and trimethoprim/sulphamethoxazole and there was no significant difference in susceptibility between isolates from chronic or sporadic infection. However, $A$. xylosoxidans isolates from chronic and sporadic cases were significantly more resistant to imipenem and ceftazidime than isolates of the outbreak clone.

Key words: Achromobacter, clinical microbiology, cystic fibrosis.

\section{INTRODUCTION}

Achromobacter spp. are opportunistic pathogens increasingly recovered from adult patients with cystic fibrosis (CF) [1]. However, owing to difficulties of accurate species identification, most clinical

\footnotetext{
* Author for correspondence: Dr E. A. Marques, Faculdade de Ciências Médicas, Disciplina de Microbiologia e Imunologia, Avenida 28 de setembro, 87, Fds, $3^{\circ}$ andar, Vila Isabel, Rio de Janeiro, RJ, 20551-030, Brazil.

(Email: marbe@uerj.br)
}

Achromobacter isolates are often reported as A. xylosoxidans. The reported prevalence of airway colonization/infection by $A$. xylosoxidans in CF centres ranges from $2 \%$ to $20 \%[2,3]$ but their clinical significance remains unclear. Nevertheless, a retrospective casecontrol study [4] showed a greater decline in lung function in patients chronically infected with A. xylosoxidans, compared to non-infected patients. The clinical course of CF lung disease is widely considered to be dependent on the nature and degree of the inflammatory response to bacterial infection and 
A. xylosoxidans has been shown to be associated with levels of inflammation similar to Pseudomonas aeruginosa in chronically infected CF patients [5].

Studies documenting the pathogenic properties of Achromobacter spp. are scarce and relatively little is known of the mechanisms which promote survival, colonization and progression to infection of the CF lung. In general, it is believed that the capability of bacteria to initiate and persist in chronic infections is due to their biofilm-forming ability, which renders them tolerant towards antimicrobial agents and host defence mechanisms [6, 7]. In addition, acquisition of antibiotic resistance and bacterial motility have been repeatedly cited as interdependent mechanisms which favour persistence of bacteria in host tissues [8-11].

On sequencing the genome of the $A$. xylosoxidans NH44784-1996 strain, Jakobsen et al. [12] identified the presence of an operon encoding an adhesin which had earlier been implicated in biofilm development by Escherichia coli [13]. Despite this suggestion of a mechanism that could promote the persistence of $A$. xylosoxidans in the CF airway, few studies have addressed the ability of Achromobacter CF isolates to form biofilms [14, 15].

We have previously reported a high prevalence $(21 \cdot 8 \%)$ of airway colonization/infection by $A$. xylosoxidans in 179 patients treated in two CF centres in Brazil, and that more than half of the patients were classed as having chronic infection [16]. Isolates were genetically heterogeneous but chronicity was associated with a relatively few of several clones identified by pulsed-field gel electrophoresis (PFGE). We also found evidence of cross-infection with the same clone in over half of all A. xylosoxidans-positive patients [16].

A. xylosoxidans is the type species of the Achromobacter genus which comprises 15 named species and multiple genogroups (http://www.bacterio. cict.fr/). Recently, new laboratory technologies have allowed the recognition of species other than $A$. xylosoxidans in CF patients, with $A$. ruhlandii being identified as the second most prevalent Achromobacter spp. in the United States [17], but by contrast, A. ruhlandii has rarely been reported from $\mathrm{CF}$ patients in Europe [18, 19].

In this study we investigated the distribution of $A$. xylosoxidans and $A$. ruhlandii in Achromobacter spp. recovered from patients treated in CF centres in Rio de Janeiro, as well as the presence of virulence traits associated with bacterial colonization of the host respiratory mucosa, such as biofilm formation, bacterial mobility and antimicrobial resistance.

\section{METHODS}

A total of 122 archived Achromobacter spp. isolates recovered over a 5-year period from 39 patients treated in two Brazilian CF centres were included in the study. All isolates had been recovered from respiratory secretions and grouped into 22 clonal types defined by PFGE [16].

Isolates were identified to species level by amplification and sequencing of gene $b l a_{\text {OXA-114-like, and by }}$ multilocus sequence type (MLST) as described previously [20]. Allelic profiles and sequence types (STs) were assigned according to the PubMLST website (http://pubmlst.org/achromobacter/).

The minimum inhibitory concentrations (MICs) of ceftazidime, ciprofloxacin, imipenem and trimetho$\mathrm{prim} / \mathrm{sulphamethoxazole} \mathrm{were} \mathrm{determined} \mathrm{with} \mathrm{E-test}$ strips (AB Biodisk, Sweden) using recommended CLSI breakpoints for non-Enterobacteriaceae [21]. $E$. coli ATCC 25922, and $P$. aeruginosa ATCC 27853 were used as quality control strains for each run of MIC determination.

Approximately $10^{7}$ c.f.u. of each isolate in $200 \mu \mathrm{l}$ Mueller-Hinton broth supplemented with $0.75 \%$ glucose were inoculated into three wells of 96-well microplates. After $20 \mathrm{~h}$ at $35 \pm 2{ }^{\circ} \mathrm{C}$ under mild agitation, the wells were washed three times with distilled water to remove non-adherent bacteria and the biofilms were stained with $200 \mu \mathrm{l}$ of $0 \cdot 1 \%$ Violet Crystal solution for $15 \mathrm{~min}$, washed, and air dried for $2 \mathrm{~h}$. The biofilm-bound stain was released with $200 \mu \mathrm{l}$ of $95 \%$ ethanol and the optical density (OD) at $595 \mathrm{~nm}$ of the obtained solutions was determined with a microplate spectrophotometric reader. Microplate wells without bacteria served as negative controls.

To assess the swimming and swarming abilities of isolates, $3 \mu \mathrm{l}$ of a fresh nutrient broth (Oxoid, UK) culture were spotted onto the centre of plates containing $0.3 \%(\mathrm{w} / \mathrm{v})$ nutrient agar, or $0.8 \%(\mathrm{w} / \mathrm{v})$ nutrient broth with $0.5 \%(\mathrm{w} / \mathrm{v})$ agar containing $0.5 \%(\mathrm{w} / \mathrm{v})$ glucose [22]. After $24 \mathrm{~h}$ at $30 \pm 1{ }^{\circ} \mathrm{C}$, the diameters of the bacterial concentric growths from the inoculation site were measured.

Data were analysed with Graph Pad Prism v. 5.0 (GraphPad Software, USA). Because two or more isolates were recovered from some individuals, we utilized median values of $\mathrm{OD}_{595}$ (biofilm) and ring 
diameters (swimming and swarming), of the different isolates from each patient. Statistical significance $(P<0.05)$ was determined by the Mann-Whitney test or by Fisher's exact test to compare antimicrobial susceptibility data.

\section{RESULTS}

Amplification and sequencing of the bla $a_{\mathrm{OXA}-114-\text { like }}$ gene and MLST of all 122 Achromobacter isolates identified $28(23.0 \%)$ isolates as $A$. ruhlandii and the remaining $94(77 \cdot 0 \%)$, as $A$. xylosoxidans. Five PFGE clonal groups were identified in $22 \mathrm{~A}$. ruhlandii isolates and 17 PFGE clonal groups in the $94 \mathrm{~A}$. xylosoxidans isolates. Seven (18.4\%) of the 39 patients harboured A. ruhlandii alone and $30(76.9 \%)$ grew only $A$. xylosoxidans; the remaining patient yielded both Achromobacter species. Four (13.3\%) of the 30 patients who harboured exclusively $A$. xylosoxidans isolates developed chronic infection which was defined as the presence of at least three positive cultures for Achromobacter spp. during a 1-year period, with a minimum 1-month interval between cultures; chronic infection was also noted in four of the seven patients harbouring $A$. ruhlandii.

The predominant 'outbreak' clone G previously defined by PFGE [16] was recovered from 22 patients and all were identified as $A$. xylosoxidans.

MLST analysis identified 13 STs in 106 isolates; 10 STs in A. xylosoxidans isolates $(n=78)$ and three STs in $A$. ruhlandii isolates $(n=28)$. We could not determine STs in 16 A. xylosoxidans isolates [PFGE clonal group $\mathrm{F}(n=11), \mathrm{J}(n=2), \mathrm{N}(n=2)$, and $\mathrm{S}(n=1)]$. Three isolates of PFGE clonal group A were further discriminated by MLST: ST13 $(n=2)$, and ST198 $(n=1)$. All clone $\mathrm{G}$ isolates fell in ST200.

Most isolates of both species were susceptible to the four antimicrobials tested and no significant differences were found in susceptibility between the species to imipenem, sulphamethoxazole/trimethoprim, and ceftazidime but $A$. ruhlandii isolates were significantly less susceptible to ciprofloxacin $(P=0 \cdot 002$, Table 1$)$. Moreover, although $A$. ruhlandii isolates from chronic infection also showed a similar susceptibility to three antimicrobials compared to sporadic isolates, there was a strong tendency for the former group towards resistance to ciprofloxacin $(P=0 \cdot 057)$. The outbreak clone of $A$. xylosoxidans exhibited significantly more resistance to ceftazidime than other isolates $(P=$ $0 \cdot 009$, Table 1) and there was a strong tendency towards resistance to imipenem for A. xylosoxidans isolates from chronic infection $(P=0 \cdot 054)$.

All isolates of both species formed biofilms on microplate wells and there was no significant difference in the degree of biofilm formation between A. xylosoxidans and $A$. ruhlandii [median $\mathrm{OD}_{595}$ $($ range $)=0.910 \quad(0 \cdot 310-1 \cdot 858)$ and $0.667 \quad(0.300-$ 1.500), respectively]. Similarly, for A. xylosoxidans, isolates from chronic or sporadic infection exhibited comparable biofilm-forming capacity [median $\mathrm{OD}_{595}$ $($ range $)=0.858 \quad(0.684-0.944)$ and $0.915 \quad(0.300$ $1 \cdot 858)$, respectively], as well as isolates of the outbreak clone compared to either chronic or sporadic infection $\left[\right.$ median OD $_{595} \quad$ (range) $=1.013 \quad(0.693-1 \cdot 217)$ and $0 \cdot 858(0 \cdot 310-1 \cdot 858)$, respectively]. Further, no significant difference in biofilm-forming capacity was evident for $A$. ruhlandii isolates from chronic or sporadic infection (median $\mathrm{OD}_{595}$ (range) $=0.895$ $(0 \cdot 300-1 \cdot 500)$ and $0 \cdot 591(0 \cdot 545-1 \cdot 106)$, respectively All isolates of both species showed a swimming phenotype but $4 \cdot 3 \%$ and $44 \cdot 4 \%$ of $A$. xylosoxidans and $A$. ruhlandii, respectively, were negative in tests for the swarming phenotype. Figure 1 shows that the swimming and swarming abilities of $A$. xylosoxidans were significantly higher than those of $A$. ruhlandii. Similarly, the mobility of isolates of the A. xylosoxidans outbreak clone was significantly higher than for other isolates of this species.

\section{DISCUSSION}

Prevalence rates of Achromobacter spp. recovered from $\mathrm{CF}$ respiratory secretions have increased in recent years. This may be due to the generally extended lifespan of these patients and the selective pressure of prolonged and multiple antimicrobial therapy. Similarly, the prevalence may be simply a consequence of increased ascertainment due to the use of improved microbiological isolation and molecular identification techniques [23] which have allowed the recognition of species other than A.xylosoxidans in the CF airway.

In an attempt to understand better the epidemiology, prevalence and level of expression of virulence traits, and antibiotic susceptibility of Achromobacter spp., 122 archived isolates from 398 patients attending two CF centers in Rio de Janeiro [16] were further investigated. Spilker et al. [17] have shown through $n r d A$ gene sequences of isolates recovered from 341 CF patients that $A$. ruhlandii accounted for $23.5 \%$ of all Achromobacter isolates, a proportion markedly 
Table 1. Distribution, MIC and rates of susceptibility to four antimicrobials in Achromobacter spp. recovered from cystic fibrosis patients

\begin{tabular}{|c|c|c|c|c|c|c|}
\hline Species (no. of isolates) & $\begin{array}{l}\text { Antimicrobial } \\
\text { agent }\end{array}$ & $\begin{array}{l}\mathrm{MIC}_{50} \\
(\mu \mathrm{g} / \mathrm{ml})\end{array}$ & $\begin{array}{l}\mathrm{MIC}_{90} \\
(\mu \mathrm{g} / \mathrm{ml})\end{array}$ & $\begin{array}{l}\text { Range } \\
(\mu \mathrm{g} / \mathrm{ml})\end{array}$ & $\begin{array}{l}\text { Susceptible } \\
(\%)\end{array}$ & $\begin{array}{l}\text { Resistant } \\
(\%)\end{array}$ \\
\hline A. xylosoxidans & IMP & 27 & 2 & $0 \cdot 25-\geqslant 32$ & $90 \cdot 4$ & $9 \cdot 6$ \\
\hline \multirow[t]{3}{*}{$(n=94)$} & SUT & 19 & 1 & $0 \cdot 032-256$ & $90 \cdot 4$ & $9 \cdot 6$ \\
\hline & CIP & 1 & 2 & $0 \cdot 38-4$ & $75 \cdot 5$ & $24 \cdot 5$ \\
\hline & CAZ & 2 & 3 & $0 \cdot 032-256$ & $88 \cdot 2$ & $11 \cdot 8$ \\
\hline A. ruhlandii & IMP & $0 \cdot 50$ & 3 & $0 \cdot 125-1$ & 100 & 0 \\
\hline \multirow[t]{3}{*}{$(n=28)$} & SUT & $0 \cdot 064$ & 32 & $0 \cdot 023-32$ & $78 \cdot 6$ & $21 \cdot 4$ \\
\hline & CIP & 2 & 4 & $0 \cdot 38-6$ & $42 \cdot 9$ & $57 \cdot 1 *$ \\
\hline & CAZ & 3 & 4 & $0 \cdot 75-12$ & 100 & 0 \\
\hline A. ruhlandii (chronic) & IMP & $0 \cdot 50$ & $0 \cdot 75$ & $0 \cdot 125-1$ & 100 & 0 \\
\hline \multirow[t]{3}{*}{$(n=22)$} & SUT & $0 \cdot 064$ & 1 & $0 \cdot 032-\geqslant 32$ & $86 \cdot 4$ & $13 \cdot 6$ \\
\hline & CIP & 2 & 4 & $0 \cdot 75-6$ & $31 \cdot 8$ & $68 \cdot 2 * *$ \\
\hline & CAZ & 3 & 4 & $0 \cdot 75-12$ & 100 & 0 \\
\hline A. ruhlandii (sporadic) & IMP & $0 \cdot 50$ & $0 \cdot 5$ & $0 \cdot 38-1$ & 100 & 0 \\
\hline \multirow[t]{3}{*}{$(n=6)$} & SUT & $0 \cdot 032$ & $\geqslant 32$ & $0 \cdot 023-\geqslant 32$ & 40 & 60 \\
\hline & CIP & $0 \cdot 38$ & 2 & $0 \cdot 38-2$ & 80 & 20 \\
\hline & CAZ & $1 \cdot 5$ & 4 & $1-4$ & 100 & 0 \\
\hline A. xylosoxidans (outbreak) & IMP & $0 \cdot 75$ & $0 \cdot 75$ & $0 \cdot 25-1 \cdot 5$ & 100 & 0 \\
\hline \multirow[t]{3}{*}{$(n=28)$} & SUT & $0 \cdot 064$ & $0 \cdot 19$ & $0 \cdot 032-10$ & $96 \cdot 4$ & $3 \cdot 6$ \\
\hline & CIP & $1 \cdot 5$ & 2 & $0 \cdot 75-3$ & $67 \cdot 0$ & $32 \cdot 1$ \\
\hline & CAZ & $1 \cdot 5$ & 4 & $0 \cdot 032-3$ & 100 & 0 \\
\hline A. xylosoxidans (chronic plus sporadic) & IMP & $0 \cdot 75$ & $\geqslant 32$ & $0 \cdot 25-\geqslant 32$ & $86 \cdot 4$ & $13 \cdot 6^{* * *}$ \\
\hline \multirow[t]{3}{*}{$(n=66)$} & SUT & $0 \cdot 125$ & $\geqslant 32$ & $0 \cdot 002-\geqslant 32$ & $87 \cdot 9$ & $12 \cdot 1$ \\
\hline & CIP & 1 & 2 & $0 \cdot 38-4$ & $78 \cdot 8$ & $21 \cdot 2$ \\
\hline & CAZ & 2 & $\geqslant 256$ & $0 \cdot 50-\geqslant 256$ & $83 \cdot 3$ & $16 \cdot 7 * * * *$ \\
\hline
\end{tabular}

MIC, Minimum inhibitory concentration; IMP, imipenem; SUT, sulphamethoxazole/trimethoprim; CIP, ciprofloxacin; CAZ, ceftazidime.

$* P=0.002: A$. ruhlandii $\times A$. xylosoxidans (CIP); $* * P=0 \cdot 057: A$. ruhlandii chronic $\times A . \quad$ ruhlandii sporadic (CIP); ${ }^{* * *} P=0 \cdot 054$ : A. xylosoxidans (chronic plus sporadic $\times$ A. xylosoxidans (outbreak) (IMP); ${ }^{* * * * P}=0 \cdot 009:$ A. xylosoxidans (chronic plus sporadic $\times A$. xylosoxidans (outbreak) (CAZ).

similar to our finding of $23.0 \%$ in the isolates reported here, and represented $21.0 \%$ of our patient cohort. Interestingly, over half $(57 \cdot 1 \%)$ of the patients harbouring $A$. ruhlandii isolates developed chronic infection compared to $13.3 \%$ in patients who grew $A$. xylosoxidans alone. However, Staphylococcus aureus and $P$. aeruginosa were isolated sporadically from three patients, but in two (who were siblings), A. ruhlandii of the same ST was the sole organism isolated before death. Although not statistically significant owing to the small number of patients studied, our findings possibly suggest that patients infected/colonized by $A$. ruhlandii are at a higher risk of developing chronic infection. It is noteworthy that a clone of Achromobacter spp. designated the Danish epidemic strain which was reported to have chronically infected 13 patients from two Danish CF centres [4, 24], was subsequently identified as $A$. ruhlandii [25].
Moreover, the species has also been implicated in causing cross-infection between $\mathrm{CF}$ patients even after limited and indirect contact between them [26], and further supports the view regarding its possible concern in $\mathrm{CF}$ communities.

Achromobacter spp. are intrinsically resistant to many antimicrobials agents, and the development of acquired resistance is common during the course of chronic infection, especially for $\beta$-lactams $[27,28]$. Moreover, variability in antimicrobial resistance of CF Achromobacter strains has been reported, although almost exclusively for $A$. xylosoxidans [14, 29]. Nevertheless, most Achromobacter isolates from our collection proved susceptible to all four antibiotics tested; the agents were chosen according to the recommendation of the CF Consensus Study Group [30]. No significant difference in susceptibility was noted between species and infection status except for the 

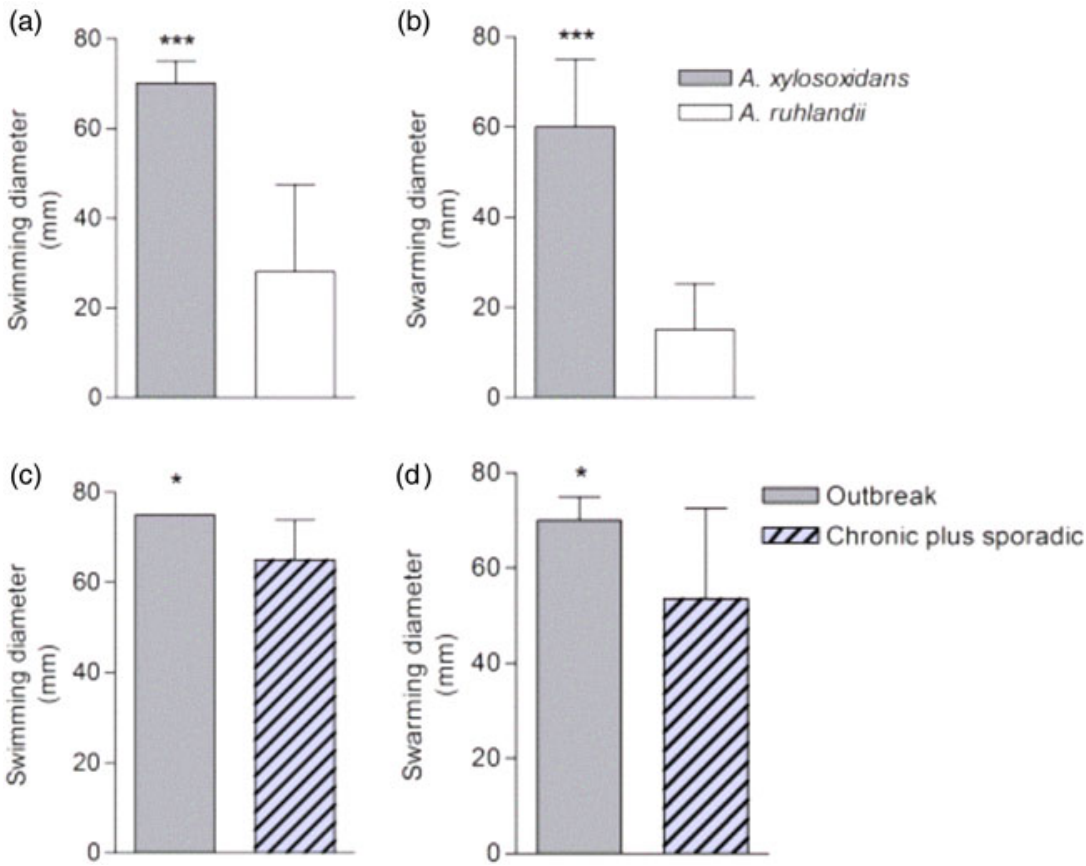

Fig. 1. (a) Swimming and (b) swarming motility of Achromobacter xylosoxidans and A. ruhlandii. (c) Swimming and (d) swarming motility of A. xylosoxidans isolates of the outbreak clone and patients with chronic or sporadic infection. Data are medians, and bars represent the interquartile range. ${ }^{*} P<0.05, * * * P<0 \cdot 001$.

outbreak clone of $A$. xylosoxidans which showed significantly increased resistance to imipenem and ceftazidime.

Adaptive mechanisms of bacteria probably explain why relatively few pathogens are able to survive and persist in the respiratory tract of CF patients despite an augmented host defence and intensive antibiotic therapy. It is well known that $P$. aeruginosa, and several other species are able to adapt to the CF host lung environment by switching to a biofilm mode of growth [31] and this property is highly correlated with the establishment of chronic infections in these patients. This present study has shown that all $A$. xylosoxidans and $A$. ruhlandii isolates produced large, and similar amounts of biofilm on an abiotic plastic surface. Although biofilm formation by $A$. xylosoxidans from $\mathrm{CF}$ patients has been previously reported, both in vivo [32] and in vitro [14], our study extends this property to $A$. ruhlandii.

Horizontal exchange of genetic material in bacteria occurs with enhanced efficiency within biofilms [8], where the dense population structure promotes plasmid dispersal through conjugation which may stimulate biofilm development. Released DNA stabilizes biofilm structure and mediates genetic exchange through transformation. Since A. xylosoxidans can serve as a reservoir of horizontal genetic transfer elements commonly involved in spreading antibiotic resistance [33], the biofilm mode of growth is likely to favour the development of multidrug resistance in CF Achromobacter.

Swimming and swarming are flagella-dependent types of bacterial motility in low-viscosity liquid and viscous environments, respectively. Most A. xylosoxidans and $A$. ruhlandii isolates studied here were shown to exhibit the swarming motility phenotype. This is in contrast to the findings of Trancassini et al. [14] who were unable to detect a swarming phenotype in 57 strains of A. xylosoxidans. Swarming has been proposed to contribute to bacterial virulence as it facilitates movement of the bacteria through the mucous layer of host epithelia and is considered to be an important means of surface epithelial colonization [34]. In some species, such as $P$. aeruginosa, swarming cells were shown to exhibit overexpression of a large number of virulence-related genes [35], whereas bacterial mutants with altered swarming motility were also defective in biofilm formation [35, 36]. Therefore, swarming is likely to be involved in early biofilm development. Finally, bacterial cells under swarming conditions have also been shown to be more resistant to the action of antibiotics than their non-swarming counterparts due to a type of adaptive resistance, and not the result of mutant selection 
$[35,37,38]$. This is particularly important because like other types of adaptive resistance, swarming would contribute to antimicrobial therapy failure even if the strains appeared susceptible by routine in vitro analysis [34]. In this study, $A$. xylosoxidans isolates, and particularly of the outbreak clone, proved to be better swarmers than chronic or sporadic isolates. This also held true for isolates of $A$. ruhlandii. It is therefore tempting to speculate that swarming mobility may be a key determinant of the ability of strains to spread through cross-infection between patients, but further research in this area is clearly warranted.

In conclusion, this investigation has provided some novel and interesting data on the relative prevalence of A. xylosoxidans and A. ruhlandii, in CF patients and the susceptibility of such isolates to antimicrobials commonly used for the treatment of these patients in our centre. Although biofilm formation and mobility mechanisms were largely similar in the isolates, an outbreak strain of $A$. xylosoxidans exhibited enhanced swarming ability and there was some correlation between this and isolates from chronic as opposed to sporadic infections. We believe that our study has given us better insight into the infectious disease process of Achromobacter in the CF lung and further investigations to clarify the relationship between the expression of bacterial virulence traits and ability to colonize/infect $\mathrm{CF}$ patients are justified.

\section{ACKNOWLEDGEMENTS}

The study was approved by the Research Ethics Committee of State University of Rio de Janeiro under number CAAE: 00716512.0.3001·5269. It was supported by the Fundação de Amparo à Pesquisa do Estado do Rio de Janeiro (FAPERJ; grant no. E-110.742/2012 and the Conselho Nacional de Desenvolvimento Científico e Tecnólogico (CNPq; grant no. 471326/2012-7), Brazil.

We thank Marcia Jones for technical support.

\section{DECLARATION OF INTEREST}

None.

\section{REFERENCES}

1. Ciofu O, Hansen CR, Høiby N. Respiratory bacterial infections in cystic fibrosis. Current Opinion in Pulmonary Medicine 2013; 19: 251-258.
2. Tan K, et al. Alcaligenes infection in cystic fibrosis. Pediatric Pulmonology 2002; 34: 101-104.

3. De Baets F, et al. Achromobacter xylosoxidans in cystic fibrosis: prevalence and clinical relevance. Journal of Cystic Fibrosis 2007; 6: 75-78.

4. Ronne-Hansen CR, et al. Chronic infection with Achromobacter xylosoxidans in cystic fibrosis patients; a retrospective case control study. Journal of Cystic Fibrosis 2006; 5: 245-251.

5. Ronne-Hansen CR, et al. Inflammation in Achromobacter xylosoxidans infected cystic fibrosis patients. Journal of Cystic Fibrosis 2010; 9: 51-58.

6. Bjarnsholt T, et al. Pseudomonas aeruginosa biofilms in the respiratory tract of cystic fibrosis patients. Pediatric Pulmonology 2009; 44: 547-558.

7. Tom SK, et al. Effect of high-dose antimicrobials on biofilm growth of Achromobacter species isolated from cystic fibrosis patients. Antimicrobial Agents and Chemotherapy 2015; 60: 650-652..

8. Molin S, Tolker-Nielsen T. Gene transfer occurs with enhanced efficiency in biofilms and induces enhanced stabilisation of the biofilm structure. Current Opinion in Biotechnology 2003; 14: 255-261.

9. Hoffman LR, et al. Aminoglycoside antibiotics induce bacterial biofilm formation. Nature 2005; 436: 1171 1175 .

10. Shrout JD, et al. The contribution of cell-cell signaling and motility to bacterial biofilm formation. MRS Bulletin 2011; 36: 367-373.

11. Boles BR, Horswill AR. Swimming cells promote a dynamic environment within biofilms. Proceedings of the National Academy of Sciences USA 2012; 109: 12848-12849.

12. Jakobsen TH, et al. Complete genome sequence of the cystic fibrosis pathogen Achromobacter xylosoxidans NH44784-1996 complies with important pathogenic phenotypes. PLoS ONE 2013; 8: e68484.

13. Wang X, Preston J, Romeo T. The pgaABCD locus of Escherichia coli promotes the synthesis of a polysaccharide adhesin required for biofilm formation. Journal of Bacteriology 2004; 186: 2724-2734.

14. Trancassini M, et al. Outbreak of Achromobacter xylosoxidans in an Italian cystic fibrosis center: genome variability, biofilm production, antibiotic resistance, and motility in isolated strains. Front Microbiology. Published online: 3 April 2014. doi:10.3389/fmicb. 2014.00138.

15. Abbott IJ, Peleg AY. Stenotrophomonas, Achromobacter, and nonmelioid Burkholderia species: antimicrobial resistance and therapeutic strategies. Seminars in Respiratory and Critical Care Medicine 2015; 36: 99-110.

16. Pereira RH, et al. Achromobacter xylosoxidans: characterization of strains in Brazilian cystic fibrosis patients. Journal of Clinial Microbiology 2011; 49: 3649-3651.

17. Spilker T, Vandamme P, Lipuma JJ. Identification and distribution of Achromobacter species in cystic fibrosis. Journal of Cystic Fibrosis 2013; 12: 298-301.

18. Coward M, et al. Use of $n r d A$ gene sequence clustering to estimate the prevalence of different Achromobacter 
species among cystic fibrosis patients in the UK. Journal of Cystic Fibrosis 2016; 15: 479-485.

19. Amoureux L, et al. Distribution of the species of Achromobacter in a French cystic fibrosis centre and multilocus sequence typing analysis reveal the predominance of $A$. xylosoxidans and clonal relationships between some clinical and environmental isolates. Journal of Cystic Fibrosis 2016; 15: 486-494.

20. Spilker T, Vandamme P, Lipuma JJ. A multilocus sequence typing scheme implies population structure and reveals several putative novel Achromobacter species. Journal of Clinial Microbiology 2012; 50: 3010-3015.

21. CLSI. Performance Standards for Antimicrobial Susceptibility Testing; Twentieth Information Supplement (January 2014 Update). CLSI, M100-S24-U. Wayne, PA: Clinical and Laboratory Standards Institute, 2014.

22. Rashid M H, Kornberg A. Inorganic polyphosphate is needed for swimming, swarming, and twitching motilities of Pseudomonas aeruginosa. Proceedings of the National Academy of Sciences USA 2000; 97: 4885-4890.

23. Lipuma JJ. The changing microbial epidemiology in cystic fibrosis. Clinical Microbiology Reviews 2010; 23: 299-323.

24. Ridderberg W, et al. Marked increase in incidence of Achromobacter xylosoxidans infections caused by sporadic acquisition from the environment. Journal of Cystic Fibrosis 2011; 10: 466-469.

25. Ridderberg W, Wang M, Nørskov-Lauritsen N. Multilocus sequence analysis of isolates of Achromobacter from patients with cystic fibrosis reveals infecting species other than Achromobacter xylosoxidans. Journal of Clinical Microbiology 2012; 50: 2688-2694.

26. Hansen CR, et al. Achromobacter species in cystic fibrosis: cross-infection caused by indirect patient-topatient contact. Journal of Cystic Fibrosis 2013; 12: 609-615.

27. Wang M, et al. Early treatment with inhaled antibiotics postpones next occurrence of Achromobacter in cystic fibrosis. Journal of Cystic Fibrosis 2013; 12: 638-643.
28. Hu Y, et al. Genomic insights into intrinsic and acquired drug resistance mechanisms in Achromobacter xylosoxidans. Antimicrobial Agents and Chemotherapy 2015; 59:1152-1161.

29. Lambiase A, et al. Achromobacter xylosoxidans respiratory tract infection in cystic fibrosis patients. European Journal of Clinical Microbiology and Infectious Disease 2011; 30: 973-980.

30. Doring G et al. Treatment of lung infection in patients with cystic fibrosis: current and future strategies. Journal of Cystic Fibrosis 2012; 11: 461-479.

31. Ciofu O, et al. Antimicrobial resistance, respiratory tract infections and role of biofilms in lung infections in cystic fibrosis patients. Advanced Drug Delivery Review 2015; 85: 7-23.

32. Hansen CR, et al. Inflammation in Achromobacter xylosoxidans infected cystic fibrosis patients. Journal of Cystic Fibrosis 2010; 9: 51-58.

33. Traglia GM, et al. Achromobacter xylosoxidans: an emerging pathogen carrying different elements involved in horizontal genetic transfer. Current Microbiology 2012; 65: 673-678.

34. Partridge JD, Harshey RM. Swarming: flexible roaming plans. Journal of Bacteriology 2013; 195: 909-918.

35. Overhage J. et al. Swarming of Pseudomonas aeruginosa is a complex adaptation leading to increased production of virulence factors and antibiotic resistance. Journal of Bacteriology 2008; 190: 2671-2679.

36. Shrout JD, et al. The impact of quorum sensing and swarming motility on Pseudomonas aeruginosa biofilm formation is nutritionally conditional. Molecular Microbiology 2006; 62: 1264-1277.

37. Kim W, et al. Swarm-cell differentiation in Salmonella enteric serovar Typhimurium results in elevated resistance to multiple antibiotics. Journal of Bacteriology 2003; 185: 3111-3117.

38. Lai S, Tremblay J, Deziel E. Swarming motility: a multicellular behaviour conferring antimicrobial resistance. Environmental Microbiology 2008; 11: 126-136. 